\section{Sensitive response to ingestion of lipids in dyspepsia}

\section{To the Editor,}

We read with interest the study published by Diella et al [1], regarding the chemical composition and texture properties of different almond varieties, and their influence on the individual sensory perception and gastrointestinal motility. The authors showed that differences in food composition including lipids might be associated with changes in gastrointestinal motility. We want to present herewith our pilot study aiming to characterize gastric accommodation and sensitive response to ingestion of lipids.

Gastric perception is initiated by the stimulation of tension receptors located in the mucosa [2]. The sensory input is modulated by various mechanisms at different levels of the gut-brain axis [3]. Lipids have been shown to sensitize mechanoreceptors response: lipid administration increases the perception of gastric distension [4]. Functional dyspeptic patients exhibit also abnormal response of the abdominal wall (diaphragmatic contraction and anterior wall relaxation) as response to meal ingestion [5].

The drink test has been developed as a noninvasive method to evaluate perception and gastric accommodation at different volume loads [6]. It turned out to be an attractive method to detect gastric accommodation dysfunctions and efficient to estimate how nutrients may influence it [7].

Starting from the hypothesis that ingestion of lipids induces a local response at gastric level (gastric accommodation) and a sensitive response (conscious sensations), we looked for these responses in relation to lipids, using the test drink.

Chronic dyspeptic patients: 15 with functional dyspepsia (FD), 11 with chronic pangastritis (CG), and 12 healthy subjects, were checked with the Short-Form Nepean Dyspepsia Index after informed consent.

We used two drinks: Drink 1: Fresubin with $6.7 \mathrm{~g} / 100 \mathrm{~mL}$ lipids content (Fresenius Kabi Deutschland $\mathrm{GmbH}$, Bad Homburg, $150 \mathrm{kcal} / 100 \mathrm{~mL}, 380 \mathrm{mOsm} / \mathrm{L}$ ) and Drink 2: Water with $0 \mathrm{~g} / 100 \mathrm{~mL}$ lipids content, as a standard to compare with. Each of the two drinks was administrated orally at a rate of $40 \mathrm{~mL} / \mathrm{min}$ until the participants reported maximum of one of the tested symptoms. Perception measurement was made every five minutes during administration and for the next 30 minutes of the recovery period, by using independent scales

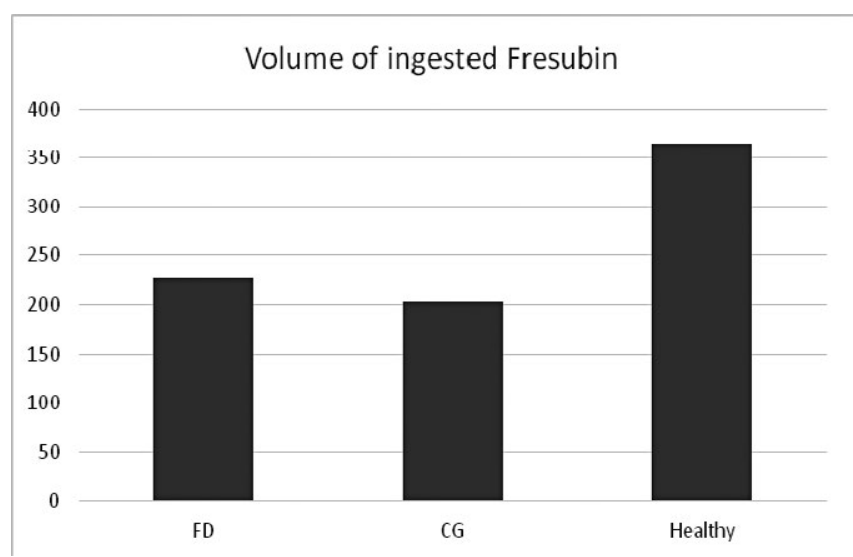

Fig. 1. Patients with functional dyspepsia (FD) tolerated lower volumes of Fresubin compared to healthy subjects $(\mathrm{p}=0.040)$, patients with chronic gastritis (CG) tolerated lower volumes of Fresubin compared to healthy subjects $(\mathrm{p}=0.004)$. There is no statistically significant difference of ingested volumes of Fresubin between patients with FD and CG.

graded for scoring: general abdominal symptoms; satiety; fullness; bloating and nausea. Results are displayed in Fig. 1.

Lipid meal ingestion induced a fullness sensation at a lower volume load in FD patients $(227.86 \pm 13.37 \mathrm{~mL})$ compared to healthy subjects $(364.17 \pm 24.49 \mathrm{~mL})$ with a statistically significant difference $(\mathrm{p}=0.040)$. The difference was also statistically significant $(\mathrm{p}=0.004)$ between the CG patients $(202.73 \pm 12 \mathrm{~mL})$ and healthy subjects $(364.17 \pm 24.49 \mathrm{~mL})$. Satiety sensation persisted longer and the recovery was slower after lipid-meal ingestion in FD $(25 \pm 0.85$ minutes $)$ with a statistically significant difference compared with healthy 
subjects $(14 \pm 1.28$ minutes $)(p=0.010)$. But the difference between CG patients ( $23 \pm 0.55$ minutes) and healthy subjects (14 \pm 1.28 minutes) was not statistically significant $(\mathrm{p}=0.101)$.

In conclusion, in both functional dyspeptic and chronic gastritis patients a lower volume lipid load was tolerated compared to healthy subjects. Our study confirmed that lipid containing meals might influence motility and symptoms of the digestive tract.

\section{Lucian-Liviu Pop, Iulia-Antonia Muresan}

$2^{\text {nd }}$ Department of Internal Medicine, Iuliu Hatieganu University of Medicine and Pharmacy, Cluj-Napoca, Romania

Correspondence: Lucian-Liviu Pop, pop.lucianliviu@yahoo.com

Conflicts of interest: None.

DOI: 10.15403/jgld.2014.1121.273.dyp

\section{REFERENCES}

1. Diella G, Di Ciaula A, Lorusso MP, et al. Distinct Effects of two Almond Cultivars on Agreeability and Gastrointestinal Motility in Healthy Subjects: more than mere Nutraceuticals. J Gastrointestin Liver Dis 2018;27:31-39. doi:10.15403/jgld.2014.1121.271.dll

2. Hernando-Harder AC, Serra J, Azpiroz F, Malagelada JR. Sites of symptomatic gas retention during intestinal lipid perfusion in healthy subjects. Gut 2004;53:661-665. doi:10.1136/gut.2003.026385

3. Kellow JE, Azpiroz F, Delvaux M, et al. Applied principles of neurogastroenterology: physiology/motility sensation. Gastroenterology 2006;130:1412-1420. doi:10.1053/j.gastro.2005.08.061

4. Serra J, Azpiroz F, Malagelada JR. Gastric distension and duodenal lipid infusion modulate intestinal gas transit and tolerance in humans. Am J Gastroenterol 2002;97:2225-2230. doi:10.1111/j.15720241.2002.05976.x

5. Burri E, Cisternas D, Villoria A, et al. Accomodation of the abdomen to its content: integrated abdomino-thoracic response. Neurogastroenterol Motil 2012;24:312-e162. doi:10.1111/j.1365-2982.2011.01846.x

6. Gonenne J, Castillo EJ, Camilleri M, et al. Does the nutrient drink test accurately predict postprandial gastric volume in health and community dyspepsia? Neurogastroenterol Motil 2005;17:44-50. doi:10.1111/j.1365-2982.2004.00588.x

7. Tack J, Caenepeel P, Piessevaux H, Cuomo R, Janssens J. Assessment of meal induced gastric accommodation by a satiety drinking test in health and in severe functional dyspepsia. Gut 2003;52:1271-1277. doi:10.1136/gut.52.9.1271

\section{Reply,}

We thank Dr. Pop and Dr. Muresan for quoting our paper recently published in JGLD [1] on the effects of two almond cultivars on agreeability and gastrointestinal motility in healthy subjects. Their letter allows us to point out some essential aspects of our study.

Our research originated because of the potential beneficial effects of nuts, including almonds (containing n-3 polynsaturated fats) on human health $[2,3]$. A recent Spanish study (PREDIMED) looked at supplementation of the Mediterranean diet with $30 \mathrm{~g}$ daily of mixed nuts ( $7.5 \mathrm{~g}$ of almonds) in people with a high cardiovascular risk. The intervention lowered total cholesterol, LDL-cholesterol and triglycerides, while decreasing the incidence of major cardiovascular events compared to subjects assigned to a reduced-fat diet [4]. We also wished to align the results of our study to the rural Apulian habit to enrich the daily "Mediterranean" diet with a specific local almond cultivar. Due to current market policy, we decided to compare the exclusive native "Filippo Cea" cultivar vs. the imported "Californian Carmel" cultivar (less expensive and widely available in supermarkets). To achieve this goal, we performed a chemical and texture analysis of almonds, and 60 subjects participated in the systematic sensory evaluation based on visual-analogue- and semi-quantitative scales to record several domains of smell, taste, chewing texture and visual perception with two almond cultivars. To describe the overall kinetic effects of almonds on the gastrointestinal tract, in another 24 additional subjects we simultaneously measured the time-dependent changes in the dilatation/ emptying of gastric antrum and gallbladder volume emptying by functional ultrasonography, and the small intestinal transit (orocecal transit time, OCTT) by H2-breath test, up to $3 \mathrm{hrs}$ following distinct meals. The standard liquid test meal $(215 \mathrm{~mL})$ consisted of $200 \mathrm{~mL}$ Nutridrink ${ }^{\circledast}$ (Nutricia, Milano, Italy) with $11.6 \mathrm{~g} \mathrm{(19 \% )} \mathrm{fat,} 300 \mathrm{kcal}$ and $15 \mathrm{~mL}$ liquid lactulose (10 g) as Lattulac $^{\circledast}$ (SOFAR, Trezzano Rosa, Milan, Italy), as previously reported by our group in a number of studies [5]. The almond mixed test meals $(215 \mathrm{~mL})$ consisted of $24 \mathrm{~g}(\mathrm{n}=12)$ almonds, $175 \mathrm{~mL}$ water and $15 \mathrm{~mL}$ lactulose with an estimated fat content of $13.5 \mathrm{~g}$ ("Filippo Cea" cultivar) and $11.8 \mathrm{~g}$ ("Californian Carmel" cultivar). Subjects ingested each meal randomly on three different days, at room temperature, and over one min. Thus, the three meals were isovolumetric and contained almost comparable amounts of fat, with a slight prevalence (i.e., 2 g) for the Filippo Cea cultivar. Indeed, Nutridrink resembles the test meal used by Pop and Muresan (200 mL Fresubin, $6.7 \mathrm{~g}$ fat $/ 100 \mathrm{~mL}$ ), used as a drink test to monitor perception and gastric accommodation at different volume loads.

In our study, we observed distinct effects of the meals with respect to gastrointestinal motility. In the stomach, both almond meals caused a similar fast and more evident antral dilatation, as compared with the liquid Nutridrink (i.e. about $14 \mathrm{~cm}^{2}$ almonds vs. $11 \mathrm{~cm}^{2}$ Nutridrink). The "mass effect" of the mixed almond meals on antral walls, likely explains this finding. Although extent and timing of gastric emptying were comparable between all meals, the overall postprandial area-under-antral emptying curve tended to be greater and emptying slower with the Filippo Cea rather than the Carmel cultivar. The slightly increased fat content in the Filippo Cea cultivar can partly explain this latter effect.

In their letter, Pop and Muresan pointed to the diagnostic value of the lipid test meal and suggested that lipid-containing meals may influence motility and symptoms of the digestive tract. In our study, perceptive features were partly different between almond cultivars. None of the isovolumetric meals in the range of 12-13.5 $\mathrm{g}$ fat, however, induced symptoms of dyspepsia, early satiety or bothering abdominal distension. This finding is likely due to the low volume employed $(215 \mathrm{~mL})$ and to the features of the study group, i.e. all healthy subjects without evidence of previous or current 
gastrointestinal disturbances. The relatively short-term duration of the follow up (3 hours), moreover, impeded a full evaluation of the satiety feeling before next meal ingestion. Pop and Muresan show that healthy subjects ingested up to $350 \mathrm{~mL}$ (=23.5 g fat), while patients with dyspepsia or chronic gastritis tolerate up to $200-220 \mathrm{~mL}$ (=13.4-14.7 g fat) Fresubin before symptoms appear. Data also confirm that both stomach and gallbladder "sense" even small differences in fat content of isovolumetric meals, and that the Filippo Cea cultivar shows the strongest effect, in this respect. In conclusion, we demonstrated that almond consumption in the range of $12-13 \mathrm{~g}$ induces evident motility responses on the stomach, gallbladder and small intestine, without inducing dyspeptic symptoms, at least in healthy volunteers. Further studies are in progress to detect if similar beneficial effects of almond meals occur in patients with distinct functional or organic gastrointestinal abnormalities.

Piero Portincasa $^{1}$, Francesco Caponio ${ }^{2}$, Maria Teresa Montagna ${ }^{3}$ 1) Clinica Medica "A. Murri”, Department of Biomedical Sciences \& Human Oncology, University of Bari Medical School, Bari; 2) Department of Soil, Plant and Food Sciences, University of Bari Aldo Moro, Bari; 3) Section of Hygiene, Department of Biomedical Science and Human Oncology, University of Bari Medical School, Bari, Italy
Correspondence: Piero Portincasa, piero.portincasa@uniba.it

Conflicts of interest: None.

DOI: 10.15403/jgld.2014.1121.273.cap

\section{REFERENCES}

1. Diella G, Di Ciaula A, Lorusso MP, et al. Distinct Effects of two Almond Cultivars on Agreeability and Gastrointestinal Motility in Healthy Subjects: more than mere Nutraceuticals. J Gastrointestin Liver Dis 2018;27:31-39. doi:10.15403/jgld.2014.1121.271.dll

2. Sabate J, Haddad E, Tanzman JS, Jambazian P, Rajaram S. Serum lipid response to the graduated enrichment of a Step I diet with almonds: a randomized feeding trial. Am J Clin Nutr 2003;77:1379-1384. doi:10.1093/ajcn/77.6.1379

3. Sabate J, Oda K, Ros E. Nut consumption and blood lipid levels: a pooled analysis of 25 intervention trials. Arch Intern Med 2010;170:821-827. doi:10.1001/archinternmed.2010.79

4. Estruch R, Ros E, Salas-Salvado J, et al. Primary Prevention of Cardiovascular Disease with a Mediterranean Diet Supplemented with Extra-Virgin Olive Oil or Nuts. N Engl J Med 2018;378:e34. doi:10.1056/ NEJMoa1800389

5. Di Ciaula A, Wang DQ, Portincasa P. Gallbladder and gastric motility in obese newborns, pre-adolescents and adults. J Gastroenterol Hepatol 2012;27:1298-1305. doi:10.1111/j.1440-1746.2012.07149.x 